\title{
Radionuclide Air Emission Report for 2008
}

\author{
Prepared by \\ Environment, Health, and Safety Division \\ Environmental Services Group
}

May 21, 2009

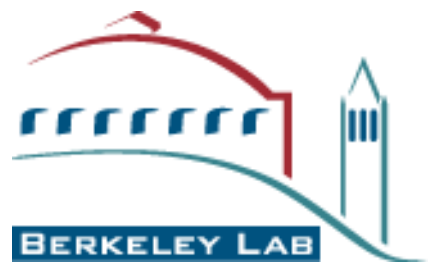

Ernest Orlando Lawrence Berkeley National Laboratory

Berkeley, CA 94720

This work was supported by the U.S. Department of Energy under Contract No. DE- AC02-05CH11231. 


\section{U.S. Department of Energy \\ Radionuclide Air Emission Report for 2008 \\ (in compliance with 40 CFR 61, Subpart H)}

Site Name: $\quad$ Ernest Orlando Lawrence Berkeley National Laboratory

Operation Office Information

Office: $\quad$ U.S. Department of Energy

Berkeley Site Office

Address: $\quad$ MS 90R1023

One Cyclotron Road

Berkeley, CA 94720

Contact: $\quad$ Kim Abbott $\quad$ Phone:(510) 486-7909

Site Information

Operator: $\quad$ University of California

Address: $\quad$ MS 85B0198

One Cyclotron Road

Berkeley, CA 94720

Contact: $\quad$ Linnea Wahl, CHP Phone:(510) 486-7623 


\section{Table of Contents}

Preface

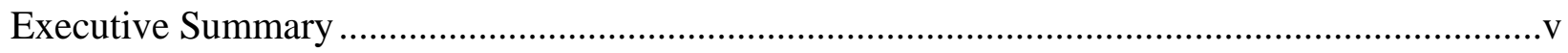

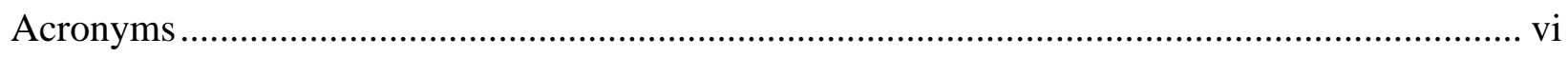

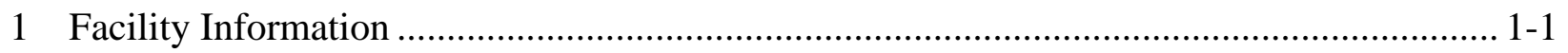

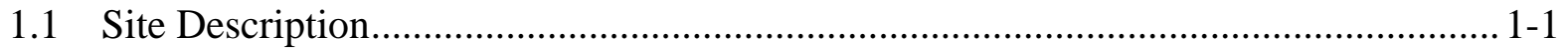

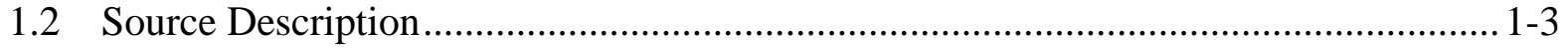

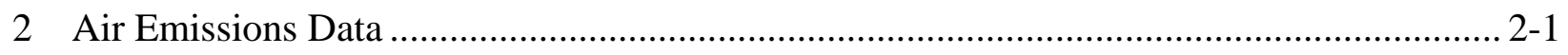

2.1 Point Sources: Measured Emissions .................................................................. 2-2

2.2 Group Sources: Calculated Emissions ................................................................. 2-4

2.3 Nonpoint Sources: Diffuse Emissions ................................................................ 2-5

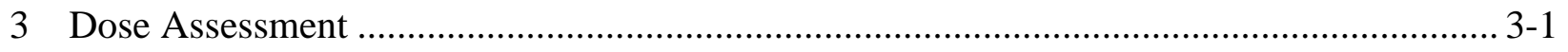

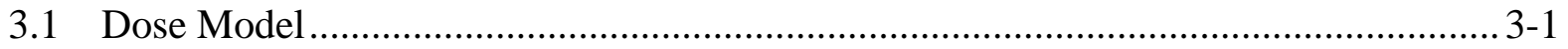

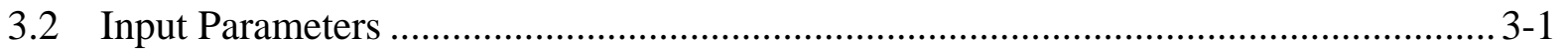

3.2.1 Building-Specific Parameters ................................................................ 3-2

3.2.2 Common Parameters ............................................................................ 3-2

3. 3 Compliance Assessment ...................................................................................... 3-3

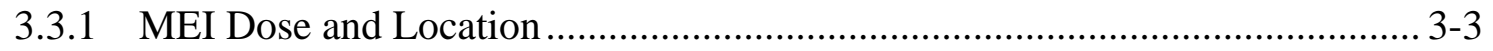

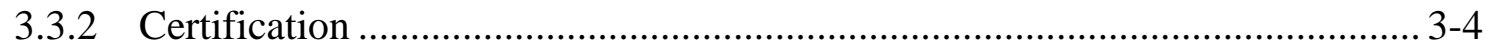

4 Additional Information ...................................................................................... 4-1

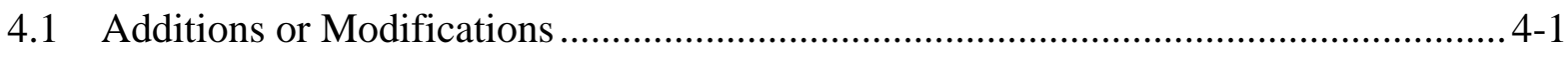

4.2 Unplanned Releases ...................................................................................... 4-1

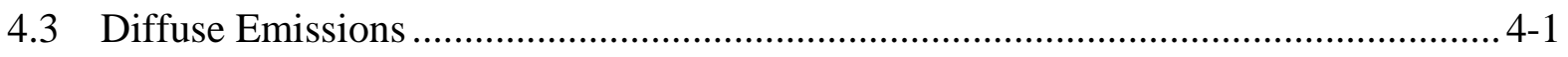

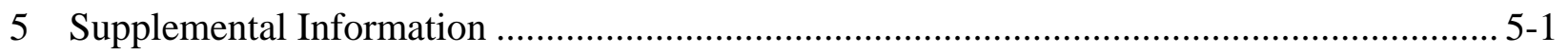

5.1 Collective Dose Estimate ............................................................................. 5-1

5.240 CFR 61 Subparts Q and T ....................................................................... 5-1

5.3 Radon Emissions.......................................................................................... 5-1

5.4 Facility Compliance ................................................................................... $5-1$

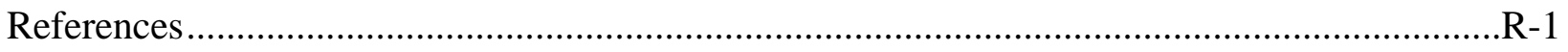

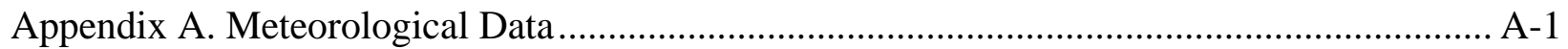

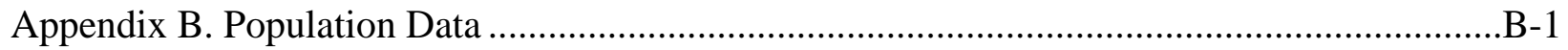




\section{LIST OF TABLES}

Table 1. Buildings Where Unsealed Radionuclide Use or Production is Authorized by

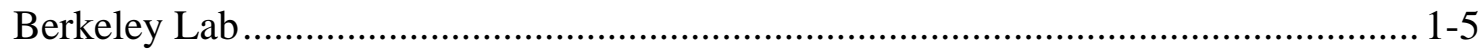

Table 2. EPA-Approved Radionuclide Emissions Measurement Approach............................. 2-1

Table 3. Measurement Category of Sources ................................................................. 2-2

Table 4. Total Activity Emitted in 2008 ........................................................................ 2-3

Table 5. Stacks Where Radionuclide Emissions are Measured ..............................................2-3

Table 6. Sources for Which Radionuclide Emissions are Calculated ......................................2-4

Table 7. Building-Specific Input Parameters ..................................................................... 3-2

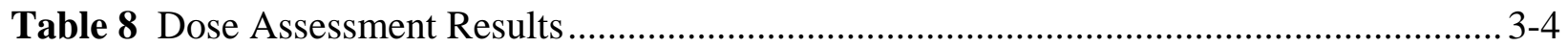

\section{LIST OF FIGURES}

Figure 1. San Francisco Bay Area Map .................................................................... 1-2

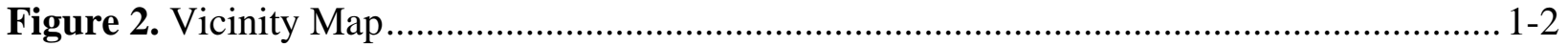

Figure 3. Berkeley Lab Buildings Where Radionuclides are Used or Produced....................... 1-4 


\section{Preface}

As a U.S. Department of Energy (DOE) facility whose operations involve the use of radionuclides, Berkeley Lab is subject to the requirements of the U.S. EPA's 40 CFR 61, National Emission Standards for Hazardous Air Pollutants (NESHAP) (EPA 1989). Subpart H of this regulation (subsequently referred to as NESHAP) establishes standards for exposure of the public to radionuclides (other than radon) released from DOE facilities. This regulation limits the emission of radionuclides to ambient air from DOE facilities. Such emissions may not exceed amounts that would cause any member of the public to receive an effective dose equivalent (subsequently referred to as dose) of $10 \mathrm{mrem} / \mathrm{yr}$ ( $0.1 \mathrm{mSv} / \mathrm{yr}$ ).

Under the NESHAP regulation, DOE facilities are required to submit an annual report each year. The NESHAP regulation specifies the content of the report and DOE provides further guidance (DOE 1994). This document is Berkeley Lab's annual report on radionuclide air emissions and meets the NESHAP requirements for reporting. This report can be found on the Laboratory's website at http://www.lbl.gov/ehs/esg/Reports/tableforreports.htm. 


\section{Executive Summary}

Berkeley Lab operates facilities where radionuclides are handled and stored. These facilities are subject to the U.S. Environmental Protection Agency (EPA) radioactive air emission regulations in Code of Federal Regulations (CFR) Title 40, Part 61, Subpart H (EPA 1989). Radionuclides may be emitted from stacks or vents on buildings where radionuclide production or use is authorized or they may be emitted as diffuse sources. In 2008, all Berkeley Lab sources were minor sources of radionuclides (sources resulting in a potential dose of less than $0.1 \mathrm{mrem} / \mathrm{yr}$ [0.001 mSv/yr]). These minor sources include more than 100 stack sources and one source of diffuse emissions. There were no unplanned emissions from the Berkeley Lab site. Emissions from minor sources (stacks and diffuse emissions) either were measured by sampling or monitoring or were calculated based on quantities used, received for use, or produced during the year. Using measured and calculated emissions, and building-specific and common parameters, Laboratory personnel applied the EPA-approved computer code, CAP88-PC, to calculate the effective dose equivalent to the maximally exposed individual (MEI). The effective dose equivalent from all sources at Berkeley Lab in 2008 is $5.2 \times 10^{-3} \mathrm{mrem} / \mathrm{yr}\left(5.2 \times 10^{-5} \mathrm{mSv} / \mathrm{yr}\right)$ to the MEI, well below the $10 \mathrm{mrem} / \mathrm{yr}$ ( $0.1 \mathrm{mSv} / \mathrm{yr})$ dose standard. The location of the MEI is at the University of California (UC) Lawrence Hall of Science, a public science museum about $1500 \mathrm{ft}$ (460 m) east of Berkeley Lab’s Building 56. The estimated collective effective dose equivalent to persons living within $50 \mathrm{mi}(80 \mathrm{~km})$ of Berkeley Lab is $1.1 \times 10^{-1}$ person-rem $\left(1.1 \times 10^{-3}\right.$ person-Sv) attributable to the Lab’s airborne emissions in 2008 . 


\section{Acronyms}

$\begin{array}{ll}\text { ALS } & \text { Advanced Light Source } \\ \text { CAP88-PC } & \text { EPA-approved dose calculation software } \\ \text { CFR } & \text { Code of Federal Regulations } \\ \text { COMPLY } & \text { EPA-approved dose calculation software } \\ \text { DOE } & \text { U. S. Department of Energy } \\ \text { EPA } & \text { U. S. Environmental Protection Agency } \\ \text { HEPA } & \text { High-efficiency particulate air } \\ \text { JBEI } & \text { Joint BioEnergy Institute } \\ \text { LHS } & \text { Lawrence Hall of Science } \\ \text { MEI } & \text { Maximally exposed individual } \\ \text { NESHAP } & \text { National Emission Standards for Hazardous Air Pollutants } \\ \text { TEDA } & \text { Triethylene diamine } \\ \text { TEDA-DAC } & \text { Triethylene-diamine-doped activated carbon } \\ \text { UC } & \text { University of California }\end{array}$




\section{Facility Information}

Lawrence Berkeley National Laboratory was founded by Ernest O. Lawrence in 1931. Lawrence received the 1939 Nobel Prize in physics for his invention of the cyclotron particle accelerator, and he is generally credited with the modern concept of interdisciplinary science, in which scientists, engineers, and technicians from different fields work together on complex scientific projects addressing national needs and programs. Lawrence's pioneering work established a great tradition of scientific inquiry and discovery at the Laboratory.

The Laboratory supports work in such diverse fields as genomics, physical biosciences, nanoscience, life sciences, fundamental physics, accelerator physics and engineering, energy conservation technology, and materials science. Through its fundamental research in these fields, Berkeley Lab has achieved international recognition for its leadership and made numerous contributions to national programs. Berkeley Lab’s research embraces the following concepts to align with the DOE mission:

- Explore the complexity of energy and matter

- Advance the science needed to attain abundant clean energy

- Understand energy impacts on our living planet

- Provide extraordinary tools for multidisciplinary research

\subsection{SITE DESCRIPTION}

Berkeley Lab is located about $3 \mathrm{mi}(5 \mathrm{~km})$ east of San Francisco Bay (see Figure 1) on land owned by UC. The Laboratory's main site is situated on approximately 202 acres (82 hectares) of this land. University of California provides long-term land leases to the DOE for the buildings at the Laboratory.

The main site lies in the hills above the UC Berkeley campus, on the ridges and draws of Blackberry Canyon (which forms the western part of the site) and adjacent Strawberry Canyon (which forms the eastern part of the site). Elevations across the site range from 450 to 1,150 ft (135 to $350 \mathrm{~m}$ ) above sea level. The western portion of the site is in Berkeley, with the eastern portion in Oakland (see Figure 2). The population of Berkeley is estimated at 100,000 and that of Oakland at 400,000 (MTC/ABAG 2000). 


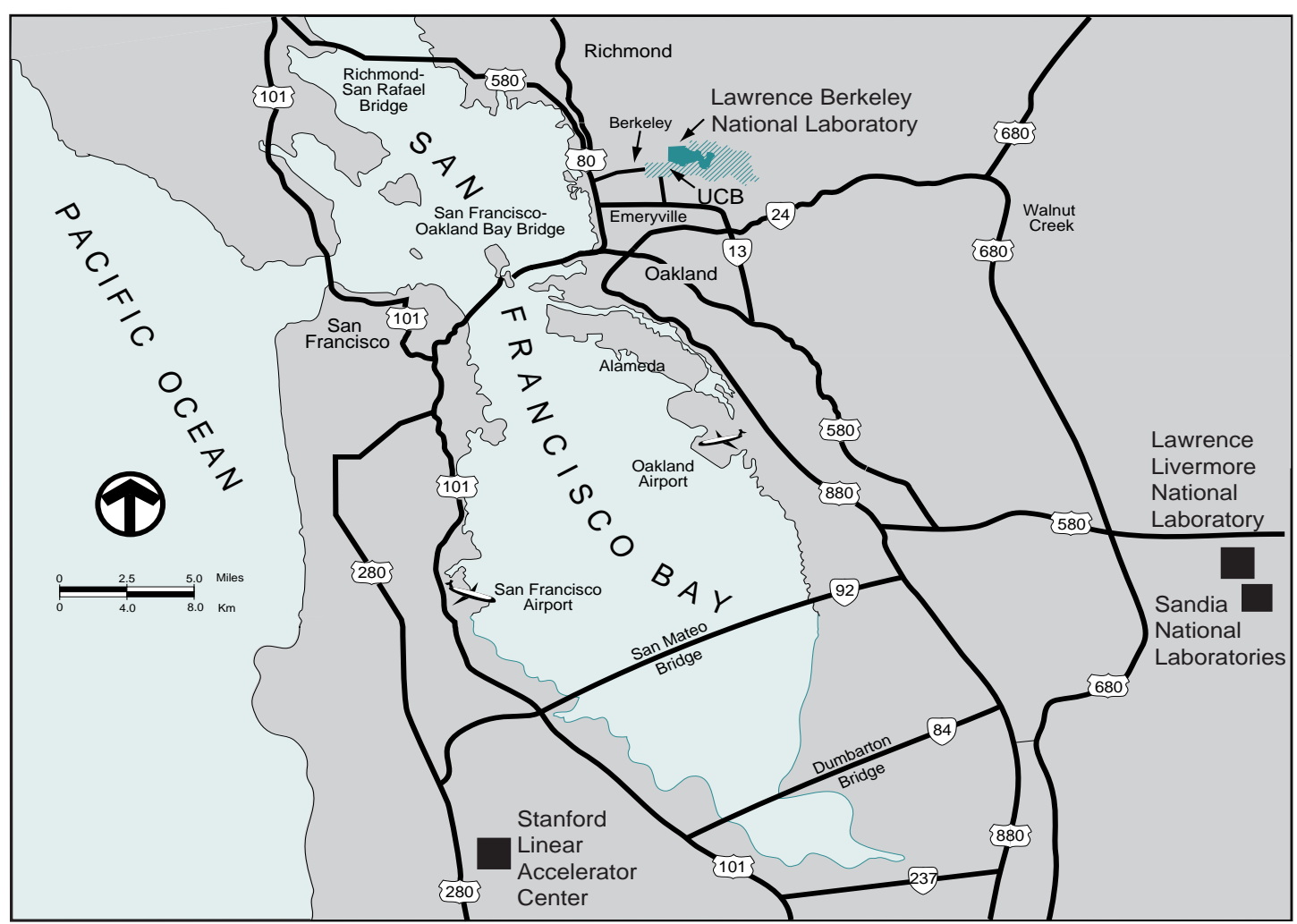

Figure 1. San Francisco Bay Area Map

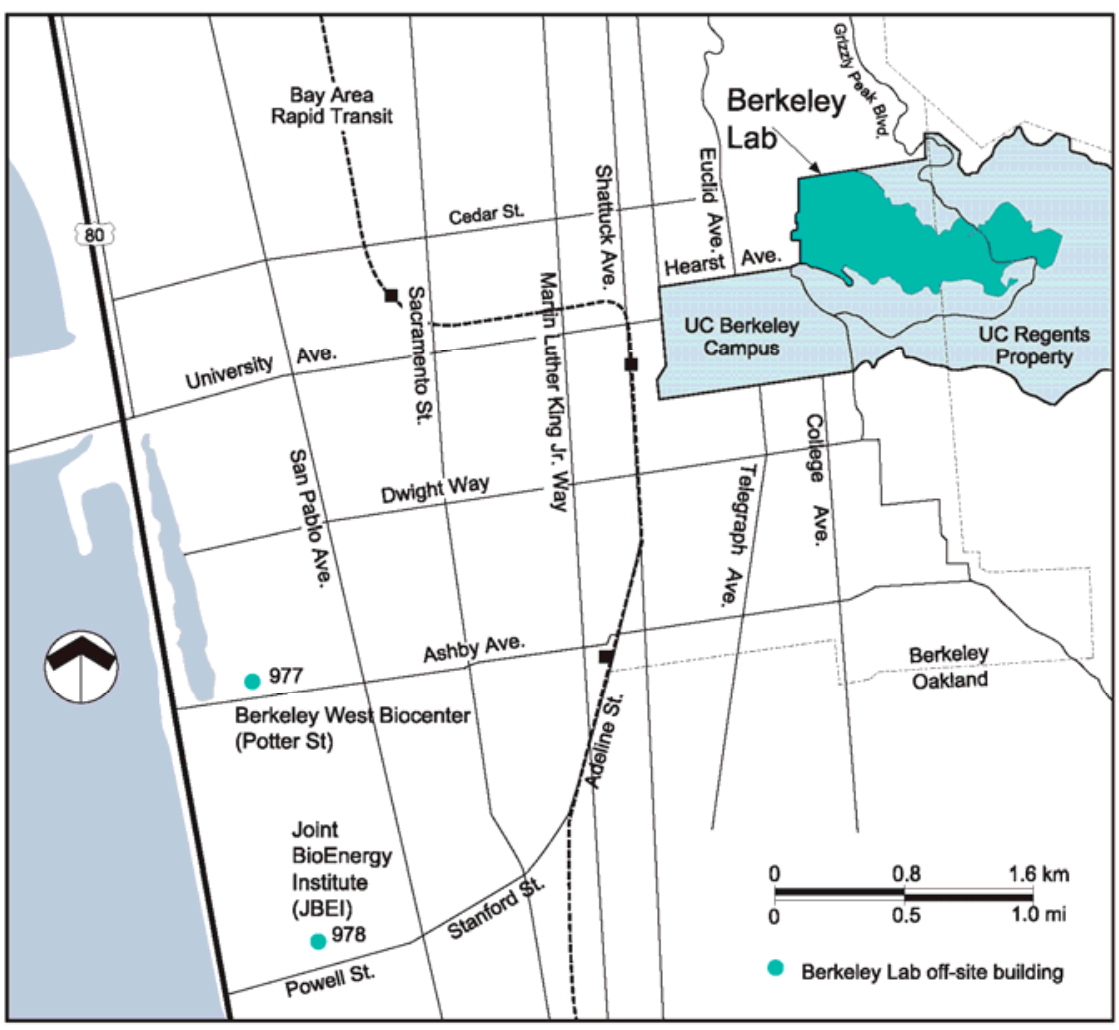

Figure 2. Vicinity Map 
Adjacent land use consists of residential, institutional, and recreational areas. The area to the south and east of the Laboratory, which is UC land, is maintained largely in a natural state but includes UC Berkeley's Strawberry Canyon Recreational Area and Botanical Garden. Northeast of the Laboratory are the university's Lawrence Hall of Science, Space Sciences Laboratory, and Mathematical Sciences Research Institute. Berkeley Lab is bordered on the north by a residential neighborhood and on the west by the UC Berkeley campus, as well as by multiunit dwellings, student residence halls, and private homes. The area to the west of Berkeley Lab is highly urbanized.

The climate of the site is temperate, influenced by the moderating effects of nearby San Francisco Bay and the Pacific Ocean to the west, and on the east by the East Bay hills paralleling the eastern shore of this same bay. These physical barriers contribute significantly to the relatively warm, wet winters and cool, dry summers of the site. In 2008, precipitation totaled 21.8 in. (55.3 cm), absolute humidity averaged $7.5 \mathrm{~g} / \mathrm{m}^{3}$, and ambient temperature averaged $55.4^{\circ} \mathrm{F}\left(13.0^{\circ} \mathrm{C}\right)$.

On-site wind patterns change little from one year to the next. The most prevalent wind pattern occurs during fair weather, with daytime westerly winds blowing off the bay, followed by lighter nighttime southeasterly drainage winds of the East Bay hills. The other predominant wind pattern is associated with storm systems passing through the region, which usually occur during the winter months. South-tosoutheast winds in advance of each storm are followed by a shift to west or northwest winds after passage of the system.

Vegetation on the Berkeley Lab site is a mixture of native plants, naturalized exotics, and ornamental species. The site was intensively grazed and farmed for approximately 150 years before the development of the Laboratory at this site in the 1930s. At the main site, the Laboratory manages on-site vegetation so that it is coordinated with the local natural succession of native plant communities. Berkeley Lab also works to maintain a wooded and savanna character in the areas surrounding buildings and roads. Ornamental species are generally restricted to public spaces and courtyards and to areas adjacent to buildings. The site has no rare, threatened, or endangered species of plants.

\subsection{SOURCE DESCRIPTION}

Berkeley Lab operates facilities subject to the EPA's NESHAP regulations where radionuclides are produced, handled, and stored (EPA 1989). Figure 3 illustrates the Berkeley Lab general site configuration, including locations of buildings where radionuclides are used or produced, the former location of Building 10 (a source of diffuse emissions in 2008), and the Lawrence Hall of Science (or LHS, the location of the MEI). Building 977, the Berkeley West Biocenter on Potter Street, and Building 978, the Joint BioEnergy Institute, are shown on Figure 2.

Researchers at the Lab use a wide variety of liquid and solid radionuclides in their research programs. Work with radioactive material may be conducted on laboratory bench tops, in fumehoods, in gloveboxes, and/or under ultra-high vacuum. In addition, radioactive gases are a by-product of charged-particle

accelerator operations in Buildings 6, 56, and 88 include ${ }^{11} \mathrm{C},{ }^{13} \mathrm{~N},{ }^{15} \mathrm{O}$, and ${ }^{18} \mathrm{~F}$, which are short-lived radionuclides. 


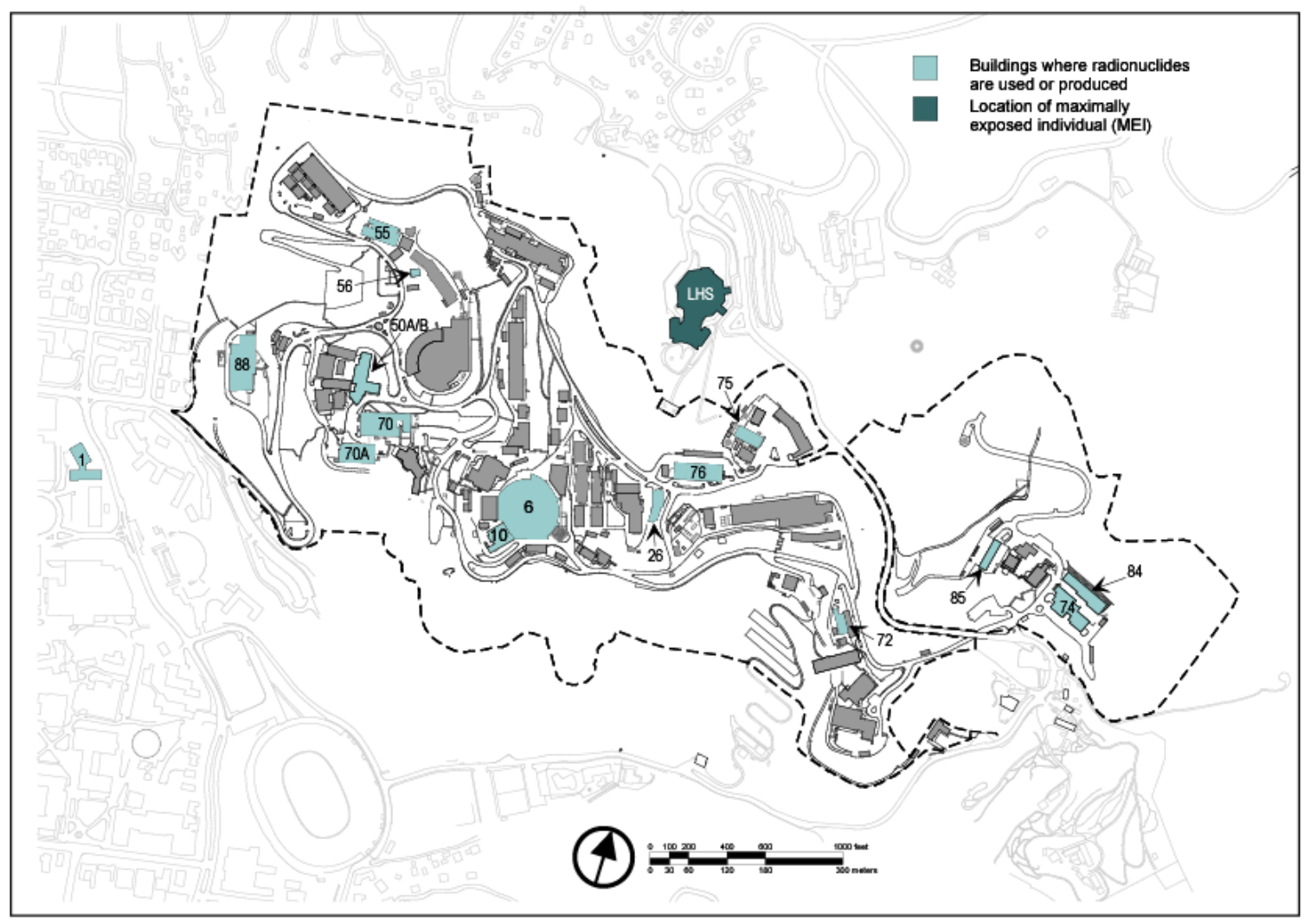

Figure 3. Berkeley Lab Buildings Where Radionuclides are Used or Produced

Radiochemical and radiobiological studies performed at Berkeley Lab typically use microcurie to tens of millicurie quantities of a variety of radionuclides. All use of radioactive material must be conducted in accordance with a Lab authorization or permit. An authorization or permit establishes the location of radioactive material areas (work areas where unsealed radioactive material is handled) and radioactive material storage areas (controlled areas where radioactive material is stored only, with no direct manipulation of the material), the required handling procedures, and appropriate work enclosures for each project.

Table 1 identifies buildings at Berkeley Lab where use or production of unsealed radioactive material was authorized in 2008 and the radionuclides that were authorized for use. Note that not all authorized radionuclides were necessarily used during the year. 
Table 1. Buildings Where Unsealed Radionuclide Use or Production is Authorized by Berkeley Lab

\begin{tabular}{|c|c|c|}
\hline Building & Building Name/ Function & Radionuclides Authorized by Berkeley Lab \\
\hline 1 & Donner Laboratory & $\mathrm{C}-14, \mathrm{H}-3, \mathrm{I}-125, \mathrm{P}-32, \mathrm{U}-238$ \\
\hline 6 & Advanced Light Source (ALS) & $\begin{array}{l}\text { Activation products, }{ }^{\mathrm{a}} \mathrm{Am}-241, \mathrm{Am}-243, \mathrm{Cm}-243, \mathrm{Cm}-246, \mathrm{Cm}-248, \mathrm{Eu}-152, \mathrm{Eu}-154, \mathrm{H}-3, \mathrm{l}-129, \mathrm{~Np}-237, \mathrm{Pu}-238, \mathrm{Pu}- \\
239, \mathrm{Pu}-240, \mathrm{Pu}-241, \mathrm{Pu}-242, \mathrm{Sr}-90, \mathrm{Tc}-99, \mathrm{Th}-232, \mathrm{U}-233, \mathrm{U}-235, \mathrm{U}-238\end{array}$ \\
\hline 26 & Radioanalytical Laboratory & $\begin{array}{l}\text { Am-241, Am-243, Cm-243, Cm-246, Cm-248, Eu-152, Eu-154, H-3, I-129, Np-237, Pu-238, Pu-239, Pu-240, Pu-241, } \\
\text { Pu-242, Sr-90, Tc-99, Th-232, U-233, U-235, U-238 }\end{array}$ \\
\hline 50A/B & Physics Research & $\begin{array}{l}\text { Ag-106m, Au-194, Co-56, Co-57, Co-58, Co-60, Mn-52, Na-22, Ni-57, Re-184m, Sc-48, Se-75, Ta-182, V-48, Zn-65, } \\
\text { Be-7, Sc-46, Ag-105, Mn-54 }\end{array}$ \\
\hline 55 & $\begin{array}{l}\text { Center for Functional } \\
\text { Imaging }\end{array}$ & $\begin{array}{l}\text { Activation products, }{ }^{a} \text { C-11, C-14, Ce-141, Co-55, Co-57, Cu-64, F-17, F-18, Ge-68, H-3, I-123, I-125, I-131, N-13, } \\
\text { Nb-95, O-14, O-15, Ru-103, Sc-46, Sr-85, Tc-99m, Tl-201 }\end{array}$ \\
\hline 56 & Biomedical Isotope Facility & Activation products, ${ }^{a} \mathrm{C}-11, \mathrm{Co}-55, \mathrm{Co}-57, \mathrm{~F}-18, \mathrm{~N}-13, \mathrm{O}-14, \mathrm{O}-15, \mathrm{~F}-17$ \\
\hline 70 & $\begin{array}{l}\text { Environmental Energy } \\
\text { Technology, Nuclear } \\
\text { Science, and Earth } \\
\text { Sciences Research }\end{array}$ & $\begin{array}{l}\text { Activation products, }{ }^{a} \text { alpha, Am-241, Am-243, Ba-133, beta-gamma, Bi-207, Ce-141, Ce-144, Cf-249, Cf-252, Cm-243, } \\
\text { Cm-248, Er-165, Er-169, Er-171, Es-253, Eu-152, Fe-59, H-3, Hf-172, Hf-175, Hf-181, Ho-166, Ho-166m, I-129, K-40, } \\
\text { Na-22, Nb-95, Np-237, P-32, Pa-233, Pu-238, Pu-239, Ra-226, Re-189, Rh-101, Sc-46, Sm-153, Sr-89, Ta-179, Ta- } \\
\text { 182, Tb-160, Tc-99, Te-125m, Th-228, Th-229, Th-230, Th-232, U-234, U-235, U-238, Yb-169, Zn-65 }\end{array}$ \\
\hline $70 \mathrm{~A}$ & $\begin{array}{l}\text { Nuclear, Chemical, and Life } \\
\text { Sciences Research }\end{array}$ & $\begin{array}{l}\text { Activation products, }{ }^{\mathrm{a}} \mathrm{Am}-241, \mathrm{Am}-243, \mathrm{Ba}-133, \mathrm{Bk}-249, \mathrm{C}-11, \mathrm{C}-14, \mathrm{Cf}-249, \mathrm{Cf}-250, \mathrm{Cf}-252, \mathrm{Cl}-36, \mathrm{Cm}-243, \mathrm{Cm}-245, \\
\mathrm{Cm}-246, \mathrm{Cm}-248, \mathrm{Co}-60, \mathrm{Es}-253 \text {, Es-254, Eu-152, Eu-154, Eu-155, Fe-59, fission products, H-3, Ni-65, Np-237, } \\
\text { Np-239, P-32, P-33, Pa-231, Pb-205, Pb-210, Pu-238, Pu-239, Pu-240, Pu-241, Pu-242, Pu-244, Ra-226, Ra-228, Ru- } \\
\text { 106, Sr-90, Tc-99, Th-228, Th-229, Th-230, Th-232, U-232, U-233, U-234, U-235, U-238, U-natural }\end{array}$ \\
\hline 72 & Low-Background Facility & $\begin{array}{l}\text { Ac-227, activation products, }{ }^{a} \text { alpha, Am-241, Au-198, Be-10, Be-7, C-11, Cf-249, Cf-252, Co-56, Co-57, Co-58, Co-60, } \\
\text { Cr-51, Cs-134, Eu-152, Eu-154, Fe-55, Fe-59, fission products, H-3, I-129, Na-22, Np-237, Np-239, P-32, Pu-238, } \\
\text { Pu-239, Sb-124, Sc-46, Se-75, Sr-90, U-238, U-238, Zn-65 }\end{array}$ \\
\hline 74 & Life Sciences Research & $\begin{array}{l}\text { C-11, C-14, Ce-141, Cu-64, F-18, Ge-68, H-3, I-123, I-125, I-131, N-13, Nb-95, O-15, P-32, Ru-103, S-35, Sc-46, Sr- } \\
\text { 85, Tc-99m, Tl-201 }\end{array}$ \\
\hline 75 & Radioanalytical Laboratory & $\begin{array}{l}\text { Ac-227, activation products, }{ }^{a} \text { alpha, Am-241, Am-243, beta-gamma, C-11, C-14, F-18, Fe-55, H-3, I-125, I-129, I-131, } \\
\text { Np-237, Pb-210, Pu-238, Pu-239, Pu-242, S-35, Sr-90, Th-229, Th-230, Th-232, U-232, U-natural }\end{array}$ \\
\hline 76 & Radioanalytical Laboratory & $\begin{array}{l}\text { Am-241, Am-243, Cm-243, Cm-246, Cm-248, Eu-152, Eu-154, H-3, I-129, Np-237, Pu-238, Pu-239, Pu-240, Pu-241, } \\
\text { Pu-242, Sr-90, Tc-99, Th-232, U-233, U-235, U-238 }\end{array}$ \\
\hline 84 & Life Sciences Research & H-3, P-32, S-35 \\
\hline 85 & $\begin{array}{l}\text { Hazardous Waste Handling } \\
\text { Facility }\end{array}$ & Alpha, beta-gamma, C-14, H-3 \\
\hline 88 & 88-Inch Cyclotron & $\begin{array}{l}\text { Ac-227, activation products, }{ }^{a} \text { actinide tracers, alpha, Am-241, Au-198, Be-7, Be-10, beta-gamma, C-11, Cf-249, Cf-252, } \\
\text { Cm-248, Co-56, Co-57, Co-58, Co-60, Cr-51, Cs-134, Eu-152, Eu-154, Fe-55, Fe-59, fission products, gamma tracers, } \\
\text { Gd-148, H-3, N-13, Na-22, Ne-18, Ne-19, Np-237, Np-239, O-14, P-32, P-33, Pb-212, Pu-238, Pu-239, Pu-242, Pu- } \\
\text { 244, Sb-124, Sc-46, Se-75, Th-228, Th-229, Th-232, U-234, U-235, U-236, U-238, U-natural, Zn-65 }\end{array}$ \\
\hline
\end{tabular}

Produced when materials such as air, water, and metals are bombarded by neutrons, protons, or other accelerated particles 
Table 1. (continued) Buildings Where Unsealed Radionuclide Use or Production is Authorized by Berkeley Lab

\begin{tabular}{|lll|}
\hline $\begin{array}{l}\text { Building } \\
977\end{array}$ & $\begin{array}{l}\text { Building Name/ Function } \\
\text { Berkeley West Biocenter } \\
\text { (Potter Street Facility) }\end{array}$ & $\begin{array}{c}\text { Radionuclides Authorized by Berkeley Lab } \\
\text { C-11, C-14, H-3, P-32, S-35 }\end{array}$ \\
978 & Joint BioEnergy Institute (JBEI) & C-14, Cd-109, H-3, P-32, S-35 \\
\hline
\end{tabular}

Produced when materials such as air, water, and metals are bombarded by neutrons, protons, or other accelerated particles 


\section{Air Emissions Data}

At Berkeley Lab, radionuclides may be emitted from stacks or other exhaust points (such as vents) on the buildings where radionuclide use is authorized (see Table 1). Alternatively, radionuclides may be uniformly released from an area or emanate from a number of points randomly distributed over an area; this is a diffuse source.

If the radionuclides emitted from any type of source could result in a potential dose of 0.1 $\mathrm{mrem} / \mathrm{yr}(0.001 \mathrm{mSv} / \mathrm{yr})$ or more to a member of the public at an off-site point where there is a residence, school, business, or office, it is considered a major source, and the EPA requires the Lab to measure its emissions continuously. Berkeley Lab has no major sources.

If emitted radionuclides could result in a potential dose of less than $0.1 \mathrm{mrem} / \mathrm{yr}(0.001 \mathrm{mSv} / \mathrm{yr})$, the source of the radionuclides is considered a minor source. The EPA requires the Lab to perform periodic confirmatory measurements on such sources. In 2008, all Berkeley Lab sources were minor sources of radionuclides. Emissions from minor sources were either measured by real-time monitoring, continuous sampling with monthly analysis of the samples, or sampling for one month at a time four times a year; or were calculated based on quantities received, used, or produced during the year.

The approach to measuring radionuclides from Berkeley Lab sources is summarized in Table 2, which EPA Region 9 approved in 2005 (Jordan 2005).

\section{Table 2. EPA-Approved Radionuclide Emissions Measurement Approach}

\begin{tabular}{|c|c|c|}
\hline $\begin{array}{l}\text { Potential Dose } \\
(\mathrm{mrem} / \mathrm{yr})^{\mathrm{a}}\end{array}$ & Category & Requirements \\
\hline dose $\geq 10.0$ & Non-compliant & $\begin{array}{l}\text { Reduction or relocation of source term and reevaluation prior } \\
\text { to authorization. }\end{array}$ \\
\hline $10.0>$ dose $\geq 1.0$ & 1 & $\begin{array}{l}\text { - Continuous sampling with weekly collection and analysis } \\
\text { AND } \\
\text { - Real-time monitoring with alarming telemetry for short- } \\
\text { lived }\left(\mathrm{t}_{1 / 2}<100 \mathrm{~h}\right) \text { radionuclides resulting in }>10 \% \text { of } \\
\text { potential dose to the maximally exposed individual. }\end{array}$ \\
\hline $1.0>$ dose $\geq 0.1$ & 2 & $\begin{array}{l}\text { - Continuous sampling with monthly collection and analysis } \\
\text { OR } \\
\text { - Real-time monitoring for short-lived }\left(\mathrm{t}_{1 / 2}<100 \mathrm{~h}\right) \\
\text { radionuclides resulting in }>10 \% \text { of potential dose to the } \\
\text { maximally exposed individual. }\end{array}$ \\
\hline $0.1>$ dose $\geq 0.01$ & 3 & Periodic sampling $25 \%$ of the year. \\
\hline dose $<0.01$ & 4 & $\begin{array}{l}\text { Potential dose evaluation before project starts and when } \\
\text { annual radionuclide use limits (as authorized by internal Lab } \\
\text { documents) are revised; no sampling or monitoring required. }\end{array}$ \\
\hline
\end{tabular}


Among the minor sources at Berkeley Lab are a few stacks, or point sources, where the emissions are measured. There are many more radioactive material areas, or group sources, where emissions are calculated.

Occasionally, activities at the Laboratory result in a diffuse source of radionuclide emissions. Diffuse sources are not actively ventilated and their emissions may be measured or calculated, depending on potential dose from those emissions (Table 2). In 2008, Berkeley Lab had one minor diffuse source, created by construction activities at the site of the former Building 10 .

A single building may have all three types of sources: point (measured stacks [typically Category 3]), group (calculated emissions [Category 4]), and diffuse (calculated wide-area emissions [Category 3 or 4]) sources (Table 3). The total activity of each radionuclide from stack air measurements and calculations is shown in Table 4.

\subsection{POINT SOURCES: MEASURED EMISSIONS}

In accordance with the EPA-approved approach (Table 2), Berkeley Lab measures emissions from stacks or other exhaust points if the potential dose from the sources could exceed 0.01 mrem/yr (0.0001 mSv/yr); these are Category 3 sources (recall that Berkeley Lab has no major [Category 1 or 2] sources). Additionally, Berkeley Lab may choose to measure emissions from stacks with less dose impact (Category 4) to ensure that those emissions are well understood. Thus stacks where emissions are measured include both Category 3 and Category 4 sources (Table 5).

At sampled stacks, a representative sample of the exhaust air passes through the appropriate collection medium (silica gel for ${ }^{3} \mathrm{H}$, sodium hydroxide solution for ${ }^{14} \mathrm{C}$, activated carbon for ${ }^{125} \mathrm{I}$, and fiberglass filter for particulate alpha- and beta-emitting radionuclides). Each medium is

Table 3. Measurement Category of Sources

\begin{tabular}{|c|c|c|c|c|c|}
\hline Building & Category 1 & Category 2 & Category 3 & Category 4 & Total \\
\hline 1 & 0 & 0 & 0 & 5 & 5 \\
\hline 6 & 0 & 0 & 0 & 9 & 9 \\
\hline 10 (diffuse) & 0 & 0 & 0 & 1 & 1 \\
\hline 26 & 0 & 0 & 0 & 4 & 4 \\
\hline $50 \mathrm{~A} / \mathrm{B}$ & 0 & 0 & 0 & 3 & 3 \\
\hline 55 & 0 & 0 & 0 & 8 & 8 \\
\hline 56 & 0 & 0 & 2 & 0 & 2 \\
\hline 70 & 0 & 0 & 0 & 10 & 10 \\
\hline $70 A$ & 0 & 0 & 2 & 22 & 24 \\
\hline 72 & 0 & 0 & 0 & 3 & 3 \\
\hline 74 & 0 & 0 & 0 & 10 & 10 \\
\hline 75 & 0 & 0 & 1 & 1 & 2 \\
\hline 76 & 0 & 0 & 0 & 1 & 1 \\
\hline 84 & 0 & 0 & 0 & 10 & 10 \\
\hline 85 & 0 & 0 & 2 & 0 & 2 \\
\hline 88 & 0 & 0 & 3 & 12 & 15 \\
\hline 977 & 0 & 0 & 0 & 4 & 4 \\
\hline 978 & 0 & 0 & 0 & 1 & 1 \\
\hline Total & 0 & 0 & 10 & 104 & 114 \\
\hline
\end{tabular}


Table 4. Total Activity Emitted in 2008

\begin{tabular}{|c|c|c|c|c|c|c|c|}
\hline $\begin{array}{l}\text { Radio- } \\
\text { nuclide }\end{array}$ & $\begin{array}{l}\text { Activity } \\
\text { (Cilyr) }^{\mathrm{a}}\end{array}$ & $\begin{array}{l}\text { Radio- } \\
\text { nuclide }\end{array}$ & $\begin{array}{l}\text { Activity } \\
\text { (Ci/yr) }\end{array}$ & $\begin{array}{l}\text { Radio- } \\
\text { nuclide }\end{array}$ & $\begin{array}{l}\text { Activity } \\
\text { (Ci/yr) }\end{array}$ & $\begin{array}{l}\text { Radio- } \\
\text { nuclide }\end{array}$ & $\begin{array}{l}\text { Activity } \\
\text { (Ci/yr) }\end{array}$ \\
\hline F-18 & $1.56 \mathrm{E}+00$ & Pu-239 & 5.43E-08 & Sr-90 & $4.27 \mathrm{E}-10$ & Th-229 & 1.83E-14 \\
\hline $\mathrm{H}-3$ & 4.23E-01 & U-238 & 5.26E-08 & $\mathrm{Ag}-108 \mathrm{~m}$ & $2.01 \mathrm{E}-10$ & $\mathrm{Na}-22$ & $9.98 \mathrm{E}-15$ \\
\hline C-11 & 1.08E-02 & Np-237 & 4.64E-08 & $\mathrm{Br}-82$ & 1.12E-10 & Cf-249 & $3.90 \mathrm{E}-15$ \\
\hline C-14 & 1.90E-03 & Pu-242 & 4.12E-08 & Co-60 & $9.55 \mathrm{E}-11$ & Cr-51 & $3.73 \mathrm{E}-15$ \\
\hline Tc-99m & 5.56E-05 & U-235 & $2.72 \mathrm{E}-08$ & Ra-226 & 7.65E-11 & Co-58 & 2.13E-15 \\
\hline P-32 & 3.08E-05 & U-234 & 1.20E-08 & Eu-155 & 6.00E-11 & Th-227 & $1.70 \mathrm{E}-15$ \\
\hline $\mathrm{N}-13$ & 1.80E-05 & Pu-241 & 1.20E-08 & Ge-75 & 5.00E-11 & Co-56 & $1.58 \mathrm{E}-15$ \\
\hline TI-201 & 4.60E-06 & Am-241 & 8.52E-09 & $\mathrm{Cm}-244$ & 2.57E-11 & $\mathrm{Hg}-194$ & $1.00 \mathrm{E}-15$ \\
\hline Ge-68 & 8.00E-07 & $\mid-125$ & 6.49E-09 & Co-57 & 1.03E-11 & Au-195 & 6.31E-16 \\
\hline $\begin{array}{l}\text { Beta } \\
\text { (Sr-90) }\end{array}$ & 6.19E-07 & Pa-231 & 4.00E-09 & I-129 & $1.00 \mathrm{E}-11$ & $\mathrm{Cm}-243$ & 3.10E-16 \\
\hline Fe-59 & $5.00 \mathrm{E}-07$ & |-131 & 3.00E-09 & Fe-55 & 8.96E-12 & Ba-133 & 2.10E-16 \\
\hline Sc-46 & 4.64E-07 & $\mathrm{Cm}-248$ & 1.67E-09 & $Z n-65$ & $8.32 \mathrm{E}-12$ & Cd-109 & $2.00 \mathrm{E}-16$ \\
\hline Ge-77 & $3.00 \mathrm{E}-07$ & Eu-152 & 1.67E-09 & Sb-125 & 8.00E-12 & Zr-88 & $1.95 \mathrm{E}-16$ \\
\hline Pu-238 & 1.85E-07 & Th-232 & 1.31E-09 & $\mathrm{Ni}-63$ & 7.58E-12 & Ag-105 & 1.86E-16 \\
\hline U-233 & 1.76E-07 & Cs-137 & 1.13E-09 & Cs-134 & 5.33E-12 & $\mathrm{Rb}-83$ & $1.05 \mathrm{E}-16$ \\
\hline $\mathrm{Am}-243$ & 1.01E-07 & Tc-99 & 1.00E-09 & Mn-54 & 3.69E-12 & $\mathrm{Be}-7$ & $2.00 \mathrm{E}-17$ \\
\hline Ar-41 & 1.00E-07 & As-76 & 1.00E-09 & Th-230 & 5.96E-13 & & \\
\hline $0-15$ & $9.40 \mathrm{E}-08$ & Cf-252 & $6.45 \mathrm{E}-10$ & Ra-228 & $6.00 \mathrm{E}-14$ & & \\
\hline $\begin{array}{l}\text { Alpha } \\
\text { (Th-232) }\end{array}$ & $6.39 \mathrm{E}-08$ & Eu-154 & $5.76 \mathrm{E}-10$ & Th-228 & 3.49E-14 & & \\
\hline
\end{tabular}

Table 5. Stacks Where Radionuclide Emissions are Measured

\begin{tabular}{|c|c|c|c|c|c|}
\hline Building & $\begin{array}{l}\text { Number of } \\
\text { Stacks }\end{array}$ & $\begin{array}{l}\text { Stack } \\
\text { Identification }\end{array}$ & $\begin{array}{l}\text { Measurement } \\
\text { Category }\end{array}$ & $\begin{array}{l}\text { Emissions } \\
\text { Control }\end{array}$ & $\begin{array}{l}\text { Efficiency } \\
(\%)\end{array}$ \\
\hline 55 & 1 & $55-128 \mathrm{H}$ & 4 & $\begin{array}{l}\text { HEPA }^{a} \\
\text { TEDA-DAC }^{b}\end{array}$ & $\begin{array}{l}>99 \\
>75\end{array}$ \\
\hline 56 & 2 & $\begin{array}{l}\text { 56-Accelerator } \\
56-\text {-Glovebox }\end{array}$ & $\begin{array}{l}3 \\
3\end{array}$ & None $^{c}$ & NA \\
\hline 70 & 1 & $70-147 A$ & 4 & HEPA & $>99$ \\
\hline $70 \mathrm{~A}$ & 2 & $\begin{array}{l}70 \mathrm{~A}-1129 \mathrm{P} / \mathrm{RT} \\
70 \mathrm{~A}-1129 \mathrm{H}\end{array}$ & $\begin{array}{l}3 \\
3\end{array}$ & HEPA & $>99$ \\
\hline 75 & 1 & $75-127 \mathrm{H}$ & 3 & HEPA & $>99$ \\
\hline 85 & 2 & $\begin{array}{l}\text { 85-Fumehood } \\
\text { 85-Glovebox }\end{array}$ & $\begin{array}{l}3 \\
3\end{array}$ & HEPA & $>99$ \\
\hline 88 & 3 & $\begin{array}{l}\text { 88-135H } \\
\text { 88-Cave0 } \\
\text { 88-RT }\end{array}$ & $\begin{array}{l}3 \\
3 \\
3\end{array}$ & HEPA & $>99$ \\
\hline
\end{tabular}

${ }^{a}$ High-efficiency particulate air filter

${ }^{\mathrm{b}}$ Triethylene-diamine-doped activated carbon trap

${ }^{\mathrm{c}}$ Radionuclides emitted from accelerators are short-lived, gaseous, activation products, for which emission control is impractical 
changed out after a month, and the radionuclides collected on the media are analyzed at a commercial laboratory. At sites that are continuously monitored in real time, a sample of the exhaust air is passed through or over detectors that provide a nearly instantaneous measurement of positron-emitting radionuclides (at Buildings 56 and 88) or alpha-emitting radionuclides (at Building 70A). Real-time measurements are recorded and archived.

Many stacks and vents at Berkeley Lab have effluent controls; that is, a filter to collect particulates or gases before they are released to the atmosphere. For example, the measured stacks (point sources) on Building 70A have high-efficiency particulate air filters to prevent small particles from entering the atmosphere. Where effluent controls are in place, samples are collected downstream from the filter. Table 5 shows effluent controls on sampled and monitored stacks.

\subsection{GROUP SOURCES: CALCULATED EMISSIONS}

In accordance with the EPA-approved approached (Table 2), Berkeley Lab calculates emissions from stacks or other exhaust points if the potential dose from the sources is less than 0.01 $\mathrm{mrem} / \mathrm{yr}(0.0001 \mathrm{mSv} / \mathrm{yr})$. These Category 4 sources (typically radioactive material areas where small amounts of radionuclides are authorized for use) are grouped by building, as shown in Table 6, to simplify reporting (DOE 1994). The amount of each radionuclide emitted is calculated by multiplying the entire quantity of that radionuclide received, used, or produced during the year by the appropriate EPA-specified release factor based on the radionuclide's physical state (provided in 40 CFR Part 61, Appendix D). This method provides a conservative, upper-bound estimate of the annual emissions.

Table 6. Sources for Which Radionuclide Emissions are Calculated

\begin{tabular}{|llll|}
\hline Building & $\begin{array}{l}\text { Number of Radioactive } \\
\text { Material Areas }\end{array}$ & Emissions Control & Efficiency (\%) \\
\hline 1 & 5 & None & NA $^{\mathrm{a}}$ \\
6 & 9 & None & NA \\
26 & 4 & None & NA \\
$50 \mathrm{~A} / \mathrm{B}$ & 3 & None & $\mathrm{NA}$ \\
55 & 8 & None & $\mathrm{NA}$ \\
70 & 10 & None & $\mathrm{NA}$ \\
$70 \mathrm{~A}$ & 22 & None & $\mathrm{NA}$ \\
72 & 3 & None & $>99$ \\
74 & 10 & HEPA & $\mathrm{NA}$ \\
75 & 1 & None & NA \\
76 & 1 & None & NA \\
84 & 10 & None & NA \\
88 & 12 & None & $>99$ \\
977 & 4 & HEPA & NA \\
978 & 1 & None & NA \\
\hline a Not applicable & None & \\
High-efficiency particulate air filter & &
\end{tabular}


Emissions are typically calculated assuming that all radionuclides received during the year are used in areas where stacks are not sampled or monitored. In fact, some received radionuclides may be emitted through sampled or monitored stacks. Emissions of these radionuclides are thus overestimated because they are accounted for as both calculated and measured emissions. For group sources there typically are no effluent controls because the emissions from these sources are very low activity.

\subsection{NONPOINT SOURCES: DIFFUSE EMISSIONS}

Berkeley Lab had one source of diffuse emissions in 2008 created by construction activities at the site of former Building 10. The soil at this site was found to be contaminated with low levels of natural uranium and was disposed of off-site at a low-level radioactive waste disposal site. Emissions from soil-handling activities were estimated using methods described in the EPA document, Methods for Estimating Fugitive Air Emissions of Radionuclides from Diffuse Sources at DOE Facilities (EPA 2004). 


\section{Dose Assessment}

\subsection{DOSE MODEL}

To comply with NESHAP regulations and DOE guidance, the EPA-approved atmospheric dispersion and radiation dose calculation computer code, CAP88-PC, Versions 2.0 and 3.0, was used to calculate the dose at various distances and from various release points (EPA 2006). For buildings 1, 977, and 978, where the nearest member of the public was much less than $328 \mathrm{ft}(100 \mathrm{~m})$ from the source, the EPAapproved dose model COMPLY was used for that location; CAP88-PC was used for doses at all other distances from the building. Doses to members of the public nearest each building were compared, and the location where the dose was greatest was determined to be the Laboratory MEI.

Dose from emissions from individual Laboratory buildings was calculated and summed for the entire facility. As identified in Figures 2 and $\underline{3}$, Buildings 1, 977 (the Berkeley West Biocenter), and 978 (the Joint BioEnergy Institute) are located outside of Berkeley Lab’s main perimeter and could be considered separate facilities since they are not on one contiguous site. However, Building 1 is located on the adjacent UC Berkeley campus and is within walking distance of the main Berkeley Lab site. Buildings 977 and 978 are located about $3 \mathrm{mi}(5 \mathrm{~km})$ southwest of the main Laboratory site. Annual radioactive air emissions from these off-site buildings and the associated dose to each nearest member of the public are much less than the highest building emissions and doses at the main Berkeley Lab site, and it would be inappropriate and misleading to model and report these much lower doses in separate reports. Therefore, for reporting and dose-modeling purposes, all off-site buildings are considered along with buildings on the Berkeley Lab site and all contribute to the potential dose received by the Laboratory MEI.

\section{$3.2 \quad$ INPUT PARAMETERS}

Input parameters to CAP88-PC include the emissions discussed in Section 2, and building-specific and common parameters, discussed below. To estimate dose, CAP88-PC, Version 3, provides a library of 825 radionuclides, which includes data for all of the radionuclides listed in Table 4 except ${ }^{252} \mathrm{Cf}$ and ${ }^{248} \mathrm{Cm}$. For these two radionuclides, Version 2 of CAP88-PC was used. (Note that the dose from ${ }^{252} \mathrm{Cf}$ and ${ }^{248} \mathrm{Cm}$ to the sitewide MEI is very low-less than $0.1 \%$ of the total dose from all radionuclides.)

In addition, when calculating dose from particulate alpha- and beta-emitting radionuclides, Berkeley Lab assigns gross alpha and gross beta measurements to the high-hazard alpha-emitting radionuclide, ${ }^{232} \mathrm{Th}$, and the high-hazard beta-emitting radionuclide, ${ }^{90} \mathrm{Sr}$, respectively. The use of the high-hazard radionuclides ${ }^{232} \mathrm{Th}$ and ${ }^{90} \mathrm{Sr}$ to represent alpha and beta emissions provides an upper-bound estimate of the dose. 


\subsubsection{Building-Specific Parameters}

For dose assessment, some Berkeley Lab buildings can be combined because they are near each other and similar operations are performed there (DOE 1994). For combined buildings and buildings with many unsampled stacks, average stack height and conservative stack diameter $(0.1 \mathrm{~m})$, exit velocity $(0 \mathrm{~m} / \mathrm{s})$, and receptor distance (from nearest edge of building) values are typically used (Table 7 ). These values overestimate the impact of air emissions on a nearby person and are chosen to ensure that stack emissions are not underestimated.

For Buildings 56 and 85, where individual stacks correlate to a single operation, the actual stack diameter and exit velocity were used and modeled separately (Stacks 1, 2, and 3). The input parameters that vary with building are shown in Table 7.

\subsubsection{Common Parameters}

The input parameters that are common among Berkeley Lab sources include meteorological data and agricultural data. Meteorological data were compiled from on-site data for 2008. Berkeley Lab collects this data from a 66- $\mathrm{ft}(20-\mathrm{m})$ tower located in the central portion of the Laboratory. Site-specific values for annual precipitation $\left(21.8 \mathrm{in}\right.$. [55.3 cm]), average ambient temperature $\left(55.4^{\circ} \mathrm{F}\left[13.0^{\circ} \mathrm{C}\right]\right)$, and average absolute humidity $\left(7.5 \mathrm{~g} / \mathrm{m}^{3}\right.$ ) were used. The default value for lid (mixing) height, $3300 \mathrm{ft}$ (1000 m), was chosen. The 2008 wind data are provided in Attachment A.

Table 7. Building-Specific Input Parameters

\begin{tabular}{|c|c|c|c|c|c|c|}
\hline $\begin{array}{l}\text { Building } \\
\text { Number }\end{array}$ & $\begin{array}{l}\text { Stack } \\
\text { Height } \\
(\mathrm{m})^{\mathrm{a}}\end{array}$ & $\begin{array}{l}\text { Stack } \\
\text { Diameter } \\
(\mathrm{m})\end{array}$ & $\begin{array}{l}\text { Exit Velocity } \\
(\mathrm{m} / \mathrm{s})\end{array}$ & $\begin{array}{l}\text { Nearest } \\
\text { Member of } \\
\text { Public }\end{array}$ & $\begin{array}{l}\text { MEI } \\
\text { Location }\end{array}$ & $\begin{array}{l}\text { Farm } \\
\text { Location }^{\text {b }}\end{array}$ \\
\hline 1 & 18 & 0.1 & 0 & $10 \mathrm{~m}$ ESE & $990 \mathrm{~m}$ ENE & $4200 \mathrm{~m} \mathrm{~N}$ \\
\hline 6 & 9 & 0.1 & 0 & 350 m NNE & $370 \mathrm{~m}$ NNE & 3200 m N \\
\hline 10 (diffuse) & 0 & $194.45^{c}$ & 0 & 350 m NNE & $370 \mathrm{~m}$ NNE & $3200 \mathrm{~m} \mathrm{~N}$ \\
\hline $26 / 76$ & 8 & 0.1 & 0 & $250 \mathrm{~m} \mathrm{~N}$ & $250 \mathrm{~m} \mathrm{~N}$ & $3200 \mathrm{~m} \mathrm{~N}$ \\
\hline $55 / 56$ & & & & $250 \mathrm{~m} \mathrm{NNW}$ & $460 \mathrm{~m} \mathrm{E}$ & $3200 \mathrm{~m} \mathrm{~N}$ \\
\hline Stack 1 & 16 & 0.3 & 3.66 & & & \\
\hline Stack 2 & 16 & 0.46 & 2.19 & & & \\
\hline Stack 3 & 12 & 0.1 & 0 & & & \\
\hline 70/70A/50A/B & 16 & 0.1 & 0 & $270 \mathrm{~m} \mathrm{WSW}$ & $530 \mathrm{~m}$ ENE & $3200 \mathrm{~m} \mathrm{~N}$ \\
\hline 72 & 3 & 0.1 & 0 & $230 \mathrm{~m} \mathrm{SSW}$ & $500 \mathrm{~m} \mathrm{NW}$ & $3200 \mathrm{~m} \mathrm{~N}$ \\
\hline $74 / 84$ & 7 & 0.1 & 0 & $160 \mathrm{~m} \mathrm{SSE}$ & $690 \mathrm{~m}$ WNW & $3200 \mathrm{~m} \mathrm{~N}$ \\
\hline 75 & 7.4 & 0.35 & 9.1 & $110 \mathrm{~m} \mathrm{NW}$ & 110 m NW & $3200 \mathrm{~m} \mathrm{~N}$ \\
\hline 85 & & & & $210 \mathrm{~m}$ SSE & $570 \mathrm{~m}$ WNW & $3200 \mathrm{~m} \mathrm{~N}$ \\
\hline Stack 1 & 16 & 0.23 & 7.24 & & & \\
\hline Stack 2 & 16 & 0.46 & 7.62 & & & \\
\hline Stack 3 & 16 & 0.1 & 0 & & & \\
\hline 88 & 13 & 0.1 & 0 & $110 \mathrm{~m} \mathrm{~W}$ & $690 \mathrm{~m}$ ENE & $3200 \mathrm{~m} \mathrm{~N}$ \\
\hline 977 & 16 & 0.1 & 0 & $30 \mathrm{~m} \mathrm{~N}$ & $5000 \mathrm{~m} \mathrm{~N}$ & $8200 \mathrm{~m} \mathrm{~N}$ \\
\hline 978 & 28 & 0.26 & 0 & $19 \mathrm{~m} \mathrm{E}$ & $5700 \mathrm{~m} \mathrm{NE}$ & $8200 \mathrm{~m} \mathrm{~N}$ \\
\hline
\end{tabular}

$1 \mathrm{~m}=3.281 \mathrm{ft}$

${ }^{\mathrm{b}}$ Approximate distance to Wildcat Canyon Regional Preserve where cattle graze

${ }^{c}$ Area of diffuse source estimated to be $194.45 \mathrm{~m}^{2}$ 
Agricultural data were obtained from the California Department of Food and Agriculture and the urban scenario was chosen (Wahl 2004). The values include the following.

- Vegetables, fraction home-produced: 0.076

- Vegetables, fraction from assessment area: 0.924

- Milk, fraction from assessment area: 1

- Meat, fraction home-produced: 0.008

- $\quad$ Meat, fraction from assessment area: 0.992

- Beef cattle density: 1.9 per $\mathrm{km}^{2}$

- Milk cattle density: 4.0 per $\mathrm{km}^{2}$

- Land fraction cultivated for vegetable crops: $4.6 \%$

\section{3 COMPLIANCE ASSESSMENT}

\subsubsection{MEI Dose and Location}

Doses from Berkeley Lab's airborne emissions are well below the $10 \mathrm{mrem} / \mathrm{yr}(0.1 \mathrm{mSv} / \mathrm{yr}) \mathrm{NESHAP}$ dose standard. As shown in Table 8, the sum of doses from all sources at Berkeley Lab in 2008 is $5.2 \times 10^{-3} \mathrm{mrem} / \mathrm{yr}\left(5.2 \times 10^{-5} \mathrm{mSv} / \mathrm{yr}\right)$ to the MEI. The location of this hypothetical person is the UC Lawrence Hall of Science, about $1500 \mathrm{ft}$ (460 m) east of Buildings 55 and 56.

Although no one actually lives at the Hall of Science, the EPA-approved software calculates the dose assuming a person resides there 24 hours a day for the entire year, eats meat and vegetables grown at the Hall of Science or nearby (see the agricultural parameters in Section 3.2.2), and drinks water from local wells contaminated with deposited airborne radionuclides. Thus the calculated dose to this hypothetical person, the MEI, is greater than the dose to an actual visitor to the Hall of Science.

Fluorine-18 emitted from Building 56 stacks accounts for about $81 \%$ of the dose to the Berkeley Lab MEI. Another $6 \%$ of the dose is due to operations with ${ }^{3} \mathrm{H}$ at the Building 85 Hazardous Waste Handling Facility, and about $4 \%$ of the dose is due to ${ }^{99}$ Tc used in Building 55 laboratories.

Annual ${ }^{18} \mathrm{~F}$ emissions from Building 56 stacks are believed to be overestimated because false-positive results occur when ${ }^{18} \mathrm{~F}$ adsorbs onto the real-time detectors. These false positive measurements are included in the calculation of annual ${ }^{18} \mathrm{~F}$ emissions. As a result, the calculated dose represents an upperbound estimate of dose from ${ }^{18} \mathrm{~F}$.

The CAP88-PC code was validated by performing a sample assessment. The output of the sample assessment was compared to output provided in the CAP88-PC, Version 3.0, users' guide (EPA 2007). The two outputs are identical, indicating that the code performed as intended. 
Table 8 Dose Assessment Results

\begin{tabular}{|c|c|c|c|}
\hline Building & $\begin{array}{l}\text { Primary Radionuclldes } \\
\text { Contributing to MEI Dose }\end{array}$ & $\begin{array}{l}\text { Dose to MEI } \\
\text { (mremiyr) }\end{array}$ & $\begin{array}{l}\text { Percent of } \\
\text { Total Dose (\%) }\end{array}$ \\
\hline 1 & P.32 & $1.3 E-7$ & $<0.1$ \\
\hline 6 & Th-232 & $2.7 \mathrm{E}-7$ & $<0.1$ \\
\hline 10 (diffuse) & $U-238$ & $4.0 \mathrm{E}-6$ & $<0.1$ \\
\hline $26 / 76$ & Np-237, Pa-231, Pu-239, Th-229 & $3.6 \mathrm{E}-5$ & 0.7 \\
\hline $55 / 56$ & $F-18, T_{C}-99^{\mathrm{C}}$ & $4.4 \mathrm{E}-3$ & 85.3 \\
\hline 70/70A/50A/B & $\mathrm{Np}-237, \mathrm{Pu}-238, \mathrm{Pu}-239$ & $3.5 \mathrm{E}-4$ & 6.8 \\
\hline 72 & Ge -77 & $6.4 \mathrm{E}-9$ & $<0.1$ \\
\hline $74 / 84$ & P-32 & $9.7 \mathrm{E}-6$ & 0.2 \\
\hline 75 & Alpha & $1.4 \mathrm{E}-5$ & 0.3 \\
\hline 85 & $\mathrm{H}-3$ & $3.1 \mathrm{E}-4$ & 6.0 \\
\hline 88 & $C-11, \cup-235, \cup-238$ & $3.8 E-5$ & 0.7 \\
\hline 977 & P-32 & $1.3 \mathrm{E}-8$ & $<0.1$ \\
\hline 978 & p-32 & $5.8 E-9$ & $<0.1$ \\
\hline Total & & $5.2 \mathrm{E}-3$ & 100 \\
\hline
\end{tabular}

Radionuclides that contribute more than $10 \%$ of the potential dose to the MEl from this source

${ }^{b}$ Dose from all radionuclides emitted; $1 \mathrm{mrem}=0.01 \mathrm{mSv}$

${ }^{\circ}$ Contributes $4 \%$ of the potential dose to the MEI from this source

\subsubsection{Certification}

I certify under penalty of law that I have personally examined and am familiar with the information submitted herein, and based on my inquiry of those individuals immediately responsible for obtaining the information, I believe that the submitted information is true, accurate, and complete. I am aware that there are significant penalties for submitting false information, including the possibility of fine and imprisonment (see 18 U.S.C. 1001).

Certified By:

Date:

Howard Hatayama, Dívision Director

EH\&S Division. Lawrence Berkeley National Lab

Certified By:

Aundra Richards, Site Manager

Date:

DOE Berkeley Site Office 


\section{Additional Information}

\subsection{ADDITIONS OR MODIFICATIONS}

There was no facility construction or modification (fabrication, erection, or installation) in 2008 relevant to the NESHAP regulation. Changes in work authorized included

- termination of radionuclide use in Building 52 and Building 64 in 2007 and

- $\quad$ authorization of radionuclide use in Building 978, the Joint BioEnergy Institute, in 2008.

\subsection{UNPLANNED RELEASES}

There were no unplanned releases in 2008 from the Berkeley Lab site.

\subsection{DIFFUSE EMISSIONS}

Berkeley Lab had one source of diffuse emissions in 2008 resulting from construction activities at the site of former Building 10. The soil at this site was found to be contaminated with low levels of natural uranium and was disposed of off-site at a low-level radioactive waste disposal site. Emissions from soilhandling activities were estimated using methods described in the EPA document, Methods for Estimating Fugitive Air Emissions of Radionuclides from Diffuse Sources at DOE Facilities (EPA 2004). The calculated dose from this diffuse source was very low, $4.0 \times 10^{-6} \mathrm{mrem} / \mathrm{y}$, which is less than $0.1 \%$ of the total dose to the Laboratory MEI. 


\section{Supplemental Information}

\subsection{COLLECTIVE DOSE ESTIMATE}

Collective population dose is calculated as the average radiation dose to a person in a specified area, multiplied by the number of people in that area. In accordance with DOE and EPA guidance documents, all radionuclides potentially emitted in 2008 (shown in Table 4) were assumed to be released from a hypothetical, centrally located stack that is $52 \mathrm{ft}(16 \mathrm{~m})$ high, is $1 \mathrm{ft}(0.3 \mathrm{~m})$ in diameter, and has an exit velocity of $13.5 \mathrm{ft} / \mathrm{s}(4.1 \mathrm{~m} / \mathrm{s})$ (Wahl 2003). The total population within $50 \mathrm{mi}(80 \mathrm{~km})$ of the Laboratory is approximately 6,615,000 based on the LandScan Global Population Database (Dobson and Bright 2002; Gallegos 2002). The population file is provided in Attachment B. The estimated collective dose to persons living within $50 \mathrm{mi}(80 \mathrm{~km})$ of Berkeley Lab is $1.1 \times 10^{-1}$ person-rem $\left(1.1 \times 10^{-3}\right.$ person-Sv) attributable to Berkeley Lab airborne emissions in 2008.

\subsection{CFR 61 SUBPARTS Q AND T}

Subparts Q and T of 40 CFR 61 are not applicable to Berkeley Lab, as the Laboratory does not operate a storage and disposal facility for radium-containing material or uranium mill tailings.

\subsection{RADON EMISSIONS}

The Laboratory does not process, manage, or possess ${ }^{232} \mathrm{U}$ or ${ }^{232} \mathrm{Th}$ in quantities that could produce an impact of $0.1 \mathrm{mrem} / \mathrm{yr}(0.001 \mathrm{mSv} / \mathrm{yr})$ or $10 \%$ of the nonradon dose to the public from ${ }^{220} \mathrm{Rn}$. The Laboratory does not maintain nondisposal or nonstorage sources of ${ }^{222} \mathrm{Rn}$ emissions in quantities that could produce an impact of $0.1 \mathrm{mrem} / \mathrm{yr}(0.001 \mathrm{mSv} / \mathrm{yr})$ or $10 \%$ of the nonradon dose to the public.

\subsection{FACILITY COMPLIANCE}

In 2008, no release points produced emissions exceeding $0.1 \mathrm{mrem} / \mathrm{yr}(0.001 \mathrm{mSv} / \mathrm{yr})$ and no sources were subject to continuous monitoring requirements. Periodic confirmatory measurements were conducted in accordance with the EPA-approved measurement approach (Table 2). 


\section{References}

DOE 1994: U.S. Department of Energy, "Calendar Year 1993 Radionuclide Air Emissions Annual Reports for DOE Sites,” memo to DOE site offices providing guidance for report preparation (March 22, 1994).

Dobson and Bright 2002: Dobson, J. E., and E. A. Bright, Landscan Global Population 1998 Database, www.ornl.gov/gist/projects/LandScan/landscan_doc.htm (August 2002).

EPA 1989: U.S. Environmental Protection Agency, National Emission Standards for Emissions of Radionuclides Other Than Radon From Department of Energy Facilities, 40 CFR Part 61, Subpart H (1989, as amended).

EPA 2004: US Environmental Protection Agency, Methods for Estimating Fugitive Air Emissions of Radionuclides from Diffuse Sources at DOE Facilities, Final Report, Eastern Research Group, Inc. (September 3, 2004).

EPA 2006: U.S. Environmental Protection Agency, National Emission Standards for Hazardous Air Pollutants (Radionuclides), Availability of Updated Compliance Model, Federal Register, Vol. 71, No. 34, p. 8854 (February 21, 2006).

EPA 2007: U.S. Environmental Protection Agency, CAP88-PC Version 3.0 User Guide, Trinity Engineering Associates, Inc. (December 2007).

Gallegos 2002: Gallegos, G., "Estimating Populations for Collective Dose Calculations,” Health Physics, Volume 83, Number 2, pages 283-286 (August 2002).

Jordan 2005: Jordan, D., "Request for Approval for LBNL to Revise Its Radionuclide NESHAP Monitoring Approach,” memo from EPA Region 9 to R. Pauer, LBNL, documenting approval of monitoring approach (April 5, 2005).

MTC/ABAG 2000: Metropolitan Transportation Commission/Association of Bay Area Governments, Bay Area Census, 2000 census data website, http://www.bayareacensus.ca.gov/cities/cities.htm (accessed April 23, 2009).

Wahl 2003: Wahl, L., “Annual Calculation of Collective Dose from Airborne Radionuclides,” memo ES03-037 to file documenting stack parameters for collective dose calculations (October 9, 2003).

Wahl 2004: Wahl, L., “Agricultural Data Used in CAP88-PC,” memo ES-05-003 to file documenting source of agricultural values used for collective dose calculations (October 26, 2004). 
Appendix A

\section{Meteorological Data}

\begin{tabular}{|c|c|c|c|c|c|c|c|}
\hline \multirow{2}{*}{$\begin{array}{c}\text { Wind } \\
\text { Direction }\end{array}$} & \multirow{2}{*}{$\begin{array}{l}\text { Stability } \\
\text { Category }\end{array}$} & \multicolumn{6}{|c|}{2008 Average Wind Frequency at Given Speed } \\
\hline & & 1-3 knots & 4-6 knots & 7-10 knots & $11-16$ knots & 17- 21 knots & $>21$ knots \\
\hline $\mathrm{N}$ & $A$ & .00148 & .00091 & .00000 & .00000 & .00000 & .00000 \\
\hline NNE & A & .00114 & .00046 & .00000 & .00000 & .00000 & .00000 \\
\hline NE & A & .00148 & .00080 & .00000 & .00000 & .00000 & .00000 \\
\hline ENE & A & .00171 & .00114 & .00000 & .00000 & .00000 & .00000 \\
\hline$E$ & A & .00114 & .00125 & .00000 & .00000 & .00000 & .00000 \\
\hline ESE & A & .00125 & .00137 & .00000 & .00000 & .00000 & .00000 \\
\hline SE & A & .00137 & .00046 & .00000 & .00000 & .00000 & .00000 \\
\hline SSE & A & .00080 & .00068 & .00000 & .00000 & .00000 & .00000 \\
\hline$S$ & A & .00228 & .00103 & .00000 & .00000 & .00000 & .00000 \\
\hline SSW & A & .00228 & .00160 & .00000 & .00000 & .00000 & .00000 \\
\hline SW & A & .00490 & .00125 & .00000 & .00000 & .00000 & .00000 \\
\hline WSW & A & .00296 & .00057 & .00000 & .00000 & .00000 & .00000 \\
\hline W & $A$ & .00444 & .00046 & .00000 & .00000 & .00000 & .00000 \\
\hline WNW & $A$ & .00262 & .00011 & .00000 & .00000 & .00000 & .00000 \\
\hline NW & $A$ & .00103 & .00171 & .00000 & .00000 & .00000 & .00000 \\
\hline NNW & A & .00091 & .00148 & .00000 & .00000 & .00000 & .00000 \\
\hline $\mathrm{N}$ & B & .00046 & .00023 & .00000 & .00000 & .00000 & .00000 \\
\hline NNE & B & .00046 & .00011 & .00000 & .00000 & .00000 & .00000 \\
\hline NE & B & .00011 & .00011 & .00023 & .00000 & .00000 & .00000 \\
\hline ENE & B & .00000 & .00068 & .00057 & .00000 & .00000 & .00000 \\
\hline$E$ & B & .00034 & .00114 & .00137 & .00000 & .00000 & .00000 \\
\hline ESE & B & .00034 & .00057 & .00000 & .00000 & .00000 & .00000 \\
\hline SE & B & .00125 & .00137 & .00023 & .00000 & .00000 & .00000 \\
\hline SSE & B & .00786 & .00649 & .00125 & .00000 & .00000 & .00000 \\
\hline $\mathrm{S}$ & B & .00581 & .00490 & .00011 & .00000 & .00000 & .00000 \\
\hline SSW & B & .00570 & .00376 & .00011 & .00000 & .00000 & .00000 \\
\hline SW & B & .00718 & .00604 & .00023 & .00000 & .00000 & .00000 \\
\hline
\end{tabular}




\begin{tabular}{|c|c|c|c|c|c|c|c|}
\hline \multirow{2}{*}{$\begin{array}{c}\text { Wind } \\
\text { Direction }\end{array}$} & \multirow{2}{*}{$\begin{array}{l}\text { Stability } \\
\text { Category }\end{array}$} & \multicolumn{6}{|c|}{2008 Average Wind Frequency at Given Speed } \\
\hline & & 1-3 knots & 4-6 knots & 7-10 knots & 11-16 knots & 17- 21 knots & $>21$ knots \\
\hline WSW & $\mathrm{B}$ & .00581 & .00775 & .00057 & .00000 & .00000 & .00000 \\
\hline W & B & .00581 & .00330 & .00023 & .00000 & .00000 & .00000 \\
\hline WNW & B & .00216 & .00057 & .00000 & .00000 & .00000 & .00000 \\
\hline NW & B & .00103 & .00194 & .00182 & .00000 & .00000 & .00000 \\
\hline NNW & $\mathrm{B}$ & .00023 & .00057 & .00011 & .00000 & .00000 & .00000 \\
\hline $\mathrm{N}$ & C & .00046 & .00011 & .00000 & .00000 & .00000 & .00000 \\
\hline NNE & C & .00011 & .00000 & .00000 & .00000 & .00000 & .00000 \\
\hline NE & C & .00011 & .00000 & .00000 & .00000 & .00000 & .00000 \\
\hline ENE & C & .00046 & .00023 & .00103 & .00080 & .00000 & .00000 \\
\hline $\mathrm{E}$ & C & .00023 & .00046 & .00205 & .00080 & .00000 & .00000 \\
\hline ESE & C & .00046 & .00023 & .00057 & .00046 & .00000 & .00000 \\
\hline SE & C & .00365 & .00570 & .00342 & .00080 & .00000 & .00000 \\
\hline SSE & C & .00433 & .00148 & .00046 & .00000 & .00000 & .00000 \\
\hline S & C & .00535 & .00273 & .00046 & .00000 & .00000 & .00000 \\
\hline SSW & C & .00365 & .00046 & .00000 & .00000 & .00000 & .00000 \\
\hline SW & C & .00399 & .00239 & .00000 & .00000 & .00000 & .00000 \\
\hline WSW & C & .01151 & .01823 & .00467 & .00000 & .00000 & .00000 \\
\hline W & C & .02017 & .02962 & .01310 & .00011 & .00000 & .00000 \\
\hline WNW & C & .01060 & .01276 & .00251 & .00046 & .00000 & .00000 \\
\hline NW & C & .00114 & .00160 & .00251 & .00000 & .00000 & .00000 \\
\hline NNW & C & .00000 & .00057 & .00023 & .00000 & .00000 & .00000 \\
\hline $\mathrm{N}$ & D & .00148 & .00262 & .00216 & .00000 & .00000 & .00000 \\
\hline NNE & D & .00057 & .00023 & .00034 & .00000 & .00000 & .00000 \\
\hline $\mathrm{NE}$ & D & .00080 & .00011 & .00023 & .00000 & .00000 & .00000 \\
\hline ENE & D & .00046 & .00046 & .00296 & .00182 & .00034 & .00000 \\
\hline$E$ & D & .00570 & .00228 & .00308 & .00273 & .00046 & .00011 \\
\hline ESE & D & .01424 & .01618 & .01401 & .00308 & .00046 & .00000 \\
\hline SE & D & .00695 & .01196 & .00980 & .00558 & .00114 & .00000 \\
\hline
\end{tabular}




\begin{tabular}{|c|c|c|c|c|c|c|c|}
\hline \multirow{2}{*}{$\begin{array}{c}\text { Wind } \\
\text { Direction }\end{array}$} & \multirow{2}{*}{$\begin{array}{l}\text { Stability } \\
\text { Category }\end{array}$} & \multicolumn{6}{|c|}{2008 Average Wind Frequency at Given Speed } \\
\hline & & 1-3 knots & 4-6 knots & 7-10 knots & 11-16 knots & 17- 21 knots & $>21$ knots \\
\hline SSE & $\mathrm{D}$ & .00194 & .00000 & .00046 & .00057 & .00000 & .00000 \\
\hline S & D & .00308 & .00011 & .00011. & .00011 & .00000 & .00000 \\
\hline SSW & $\mathrm{D}$ & .00148 & .00000 & .00023 & .00000 & .00000 & .00000 \\
\hline SW & D & .00148 & .00000 & .00000 & .00000 & .00000 & .00000 \\
\hline WSW & D & .00570 & .00103 & .00091 & .00011 & .00000 & .00000 \\
\hline W & $\mathrm{D}$ & .02609 & .01025 & .00524 & .00034 & .00000 & .00000 \\
\hline WNW & $\mathrm{D}$ & .02199 & .02245 & .01436 & .00319 & .00011 & .00000 \\
\hline NW & D & .01253 & .01276 & .00479 & .00000 & .00000 & .00000 \\
\hline NNW & D & .00456 & .00968 & .00923 & .00000 & .00000 & .00000 \\
\hline$N$ & $E$ & .00148 & .00194 & .00034 & .00000 & .00000 & .00000 \\
\hline NNE & $E$ & .00160 & .00034 & .00000 & .00000 & .00000 & .00000 \\
\hline NE & E & .00080 & .00011 & .00000 & .00000 & .00000 & .00000 \\
\hline ENE & $E$ & .00125 & .00068 & .00046 & .00000 & .00000 & .00000 \\
\hline$E$ & $E$ & .00615 & .00125 & .00068 & .00000 & .00000 & .00000 \\
\hline ESE & $E$ & .00980 & .00592 & .00068 & .00000 & .00000 & .00000 \\
\hline SE & $E$ & .01037 & .00695 & .00023 & .00000 & .00000 & .00000 \\
\hline SSE & $E$ & .00342 & .00000 & .00023 & .00000 & .00000 & .00000 \\
\hline $\mathrm{S}$ & $\bar{E}$ & .00034 & .00000 & .00000 & .00000 & .00000 & .00000 \\
\hline SSW & $\bar{E}$ & .00011 & .00000 & .00000 & .00000 & .00000 & .00000 \\
\hline SW & $\bar{E}$ & .00011 & .00000 & .00011 & .00000 & .00000 & .00000 \\
\hline WSW & $\bar{E}$ & .00091 & .00251 & .00023 & .00000 & .00000 & .00000 \\
\hline W & $E$ & .00285 & .00798 & .00023 & .00000 & .00000 & .00000 \\
\hline WNW & $E$ & .00809 & .00444 & .00023 & .00000 & .00000 & .00000 \\
\hline NW & $E$ & .01037 & .00399 & .00011 & .00000 & .00000 & .00000 \\
\hline NNW & $E$ & .00467 & .00752 & .00205 & .00000 & .00000 & .00000 \\
\hline $\mathrm{N}$ & $F$ & .01071 & .00262 & .00000 & .00000 & .00000 & .00000 \\
\hline NNE & $F$ & .00501 & .00103 & .00000 & .00000 & .00000 & .00000 \\
\hline NE & $F$ & .00661 & .00091 & .00000 & .00000 & .00000 & .00000 \\
\hline
\end{tabular}




\begin{tabular}{|c|c|c|c|c|c|c|c|}
\hline Wind & Stability & \multicolumn{7}{|c|}{ 2008 Average Wind Frequency at Given Speed } \\
\cline { 3 - 8 } Direction & Category & 1-3 knots & 4-6 knots & 7-10 knots & 11-16 knots & 17- 21 knots & > 21 knots \\
\hline ENE & F & .00524 & .00194 & .00000 & .00000 & .00000 & .00000 \\
E & F & .01344 & .00353 & .00023 & .00000 & .00000 & .00000 \\
ESE & F & .01937 & .00160 & .00000 & .00000 & .00000 & .00000 \\
SE & F & .02245 & .00809 & .00000 & .00000 & .00000 & .00000 \\
SSE & F & .02643 & .00672 & .00034 & .00000 & .00000 & .00000 \\
S & F & .01367 & .00296 & .00011 & .00000 & .00000 & .00000 \\
SSW & F & .01253 & .00228 & .00011 & .00000 & .00000 & .00000 \\
SW & F & .01390 & .00513 & .00000 & .00000 & .00000 & .00000 \\
WSW & F & .01276 & .00991 & .00000 & .00000 & .00000 & .00000 \\
W & F & .01561 & .00444 & .00000 & .00000 & .00000 & .00000 \\
WNW & F & .01527 & .00171 & .00000 & .00000 & .00000 & .00000 \\
NW & F & .01812 & .00330 & .00011 & .00000 & .00000 & .00000 \\
NNW & F & .01413 & .00376 & .00000 & .00000 & .00000 & .00000 \\
\hline
\end{tabular}


Appendix B

\section{Population Data}

\begin{tabular}{|c|c|c|c|c|c|c|c|c|c|c|c|c|c|}
\hline \multirow[t]{2}{*}{ Direction } & \multicolumn{13}{|c|}{ Population at Given Distance from Center from Berkeley Lab } \\
\hline & 0.5 km & $1 \mathrm{~km}$ & $2 \mathrm{~km}$ & $3 \mathrm{~km}$ & $4 \mathrm{~km}$ & $5 \mathrm{~km}$ & $10 \mathrm{~km}$ & $20 \mathrm{~km}$ & 30 km & $40 \mathrm{~km}$ & 50 km & 60 km & 80 km \\
\hline$N$ & 0 & 0 & 419 & 0 & 0 & 0 & 359 & 30398 & 98105 & 32491 & 68070 & 24776 & 1797 \\
\hline NNW & 0 & 0 & 808 & 2443 & 2430 & 2793 & 2484 & 52319 & 0 & 993 & 11808 & 26337 & 116202 \\
\hline NW & 0 & 2291 & 1278 & 1627 & 3649 & 3453 & 36003 & 78123 & 1753 & 43472 & 15539 & 61782 & 148052 \\
\hline WNW & 0 & 0 & 3813 & 6632 & 7002 & 7163 & 22596 & 15684 & 91680 & 35109 & 2452 & 2444 & 987 \\
\hline w & 0 & 0 & 0 & 6789 & 6941 & 3885 & 14 & 6636 & 45790 & 1780 & 588 & 0 & 0 \\
\hline WSW & 0 & 0 & 4181 & 2416 & 7039 & 2982 & 24 & 158141 & 158570 & 0 & 0 & 0 & 0 \\
\hline SW & 0 & 4530 & 4402 & 2967 & 7448 & 8834 & 5707 & 123614 & 429877 & 77558 & 8 & 0 & 0 \\
\hline SSW & 0 & 0 & 3345 & 1553 & 8699 & 5591 & 51318 & 12682 & 26539 & 143163 & 20902 & 1540 & 218 \\
\hline S & 0 & 0 & 2912 & 2404 & 6515 & 2818 & 107157 & 64082 & 0 & 120732 & 209651 & 39481 & 6338 \\
\hline SSE & 0 & 1270 & 1096 & 716 & 3089 & 3224 & 41486 & 188837 & 171923 & 161075 & 158439 & 272632 & 1153570 \\
\hline SE & 0 & 0 & 0 & 1122 & 796 & 1867 & 11308 & 12453 & 74898 & 49162 & 77674 & 9220 & 45859 \\
\hline ESE & 0 & 0 & 51 & 189 & 0 & 0 & 4648 & 11966 & 64845 & 64822 & 69656 & 8301 & 38140 \\
\hline E & 0 & 0 & 0 & 19 & 130 & 37 & 8755 & 61557 & 15866 & 2791 & 21010 & 12024 & 42996 \\
\hline ENE & 0 & 0 & 40 & 2 & 98 & 1982 & 6467 & 76620 & 121897 & 96518 & 87328 & 10455 & 12356 \\
\hline NE & 0 & 0 & 0 & 0 & 22 & 17 & 908 & 46933 & 19832 & 6565 & 727 & 1675 & 1447 \\
\hline NNE & 346 & 0 & 0 & 0 & 0 & 6 & 19 & 5603 & 28838 & 7625 & 99040 & 93357 & 42715 \\
\hline
\end{tabular}

